\title{
LACK OF RECOMBINATION BETWEEN X CHROMOSOMES IN INTERSPECIFIC HYBRIDS OF THE ANOPHELES GAMBIAE GROUP OF SPECIES
}

\author{
CHRISTOPHER A. GREEN \\ Department of Medical Entomology, South African Institute for Medical Researeh, \\ P.O. Box 1038, Johannesburg 2000
}

Received 21.xii.79

Curtis and Ghalkley (1979) suggest that asynapsis in polytene chromosomes, particularly in the sex chromosomes, in $F_{1}$ interspecific hybrids between Anopheles gambiae, $A$. arabiensis, and $A$. quadriannulatus, might be indicative of asynapsis during meiosis in these same hybrids. If this is true, they argue, then crossing over would most likely not occur in hybrids during meiosis. They then present data which they consider tests "these expectations with regard to the X chromosome".

The data consist of breeding experiments between a strain of $A$. gambiae carrying a sex-linked mutant, and strains of $A$. arabiensis and $A$. quadriannulatus which are wild-type with respect to the mutant marker. Each species has a distinct $\mathrm{X}$ chromosome. In numerous back-cross progeny from the $\mathrm{F}_{1}$ hybrids $\times A$. gambiae males, no recombinants were recovered, i.e., the sex-linked mutant only and always occurred when the gambiae type $\mathrm{X}$ chromosome was homozygous (the mutant is recessive). The authors conclude from these results that it is likely that there is no pairing or crossing over between the $\mathrm{X}$ chromosomes at meiosis in female hybrids. However, it is probable that neither of these conclusions is valid from the data.

Curtis and Chalkley (1979) point out that these species differences in the $\mathrm{X}$ chromosomes " can be largely explained by postulating a series of inversions". Figure 1 shows a diagram of the X chromosome of $A$. quadriannulatus with the break-points of these inversions, and the specific inversion formulae for this arm for each species.

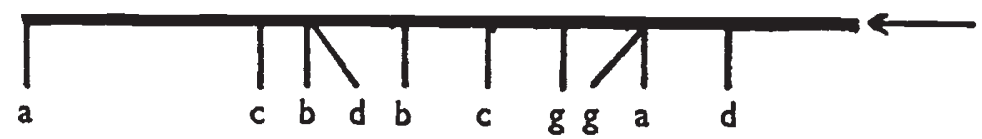

A. gambiae Xag, A. arabiensis Xbcd

Fig. 1.-A diagram of the $\mathrm{X}$ chromosome of $A$. quadriannulatus showing the association of interspecific, fixed inversions between it and $A$. gambiae and $A$. arabiensis (after Coluzzi and Sabatini, 1968; Coluzzi et al., 1979). The arrow indicates the centromeric end of the arm.

$\mathrm{F}_{1}$ interspecific hybrids between $A$. gambiae and $A$. arabiensis will be heterozygous for Xagbcd which involves about 85 per cent of the arm, and, between gambiae and quadriannulatus, heterozygous for Xag involving about 75 per cent of the arm. We do not know where the mutant locus is located 
along the X. Most of the back-cross individuals of Curtis and Chalkley (1979) were between gambiae and arabiensis $(\mathrm{n}=134)$, and very few between gambiae and quadriannulatus $(\mathrm{n}=17)$. It is highly unlikely that any crossovers in the heterozygotes for Xagbcd would result in viable gametes within the inversion complex, and those outside it would not be detected unless the mutant locus lay in this 15 per cent of the arm. In fact the actual portion available for recoverable recombinants would probably be less, due to the well known effect of paracentric inversions on chromosome segments lying immediately outside inversion loops. It might be argued that recombination in the terminal, centromeric segment would have been detectable, as synapsis might be expected where a terminal piece of a gambiae $\mathrm{X}$ had been recombined with an arabiensis $\mathrm{X}$, but it is a tenuous expectation at present.

It seems reasonable to suggest that the questions posed by Curtis and Chalkley (1979) concerning asynapsis and no crossing over in $F_{1}$ hybrids can only be answered in the absence of other variables which are known to have a powerful effect on the phenomenon under test. Their projected work on the homosequential autosomes of these species would seem to meet this requirement.

\section{REFERENCES}

COluzzi, M., AND SABATinI, A. 1968. Cytogenetic observations on species C of the Anopheles gambiae complex. Parassitologia, 10, 155-165.

Coluzzi, M., SABAtini, A., Petrarca, v., AND di DECO, M. A. 1979. Chromosomal differentiation and adaptation to human environments in the Anopheles gambiae complex. Trans. Roy. Soc. Trop. Hyg., 73, 483-497.

CURTIS, C., AND CHALKLEY, J. 1979. Lack of recombination between the X chromosomes of different members of the Anopheles gambiae complex. Heredity, 42, 323.326. 